\title{
Spreading dynamics and contact angle of completely wetting volatile drops
}

\author{
Etienne Jambon-Puillet ${ }^{1} \dagger$, Odile Carrier ${ }^{1}$, Noushine Shahidzadeh ${ }^{1}$, \\ David Brutin ${ }^{2}$, Jens Eggers ${ }^{3}$ and Daniel Bonn ${ }^{1}$ \\ ${ }^{1}$ Institute of Physics, Van der Waals-Zeeman Institute, University of Amsterdam, Science Park \\ 904, Amsterdam, the Netherlands \\ ${ }^{2}$ Aix-Marseille University, IUSTI UMR 7343, 13453 Marseille, France \\ ${ }^{3}$ School of Mathematics - University of Bristol, University Walk, Bristol BS8 1TW, United \\ Kingdom
}

(Received xx; revised xx; accepted xx)

The spreading of evaporating drops without a pinned contact line is studied experimentally and theoretically, measuring the radius $R(t)$ of completely wetting alkane drops of different volatility on glass. Initially the drop spreads ( $R$ increases), then owing to evaporation reverses direction and recedes with an almost constant non-zero contact angle $\theta \propto \beta^{1 / 3}$, where $\beta$ measures the rate of evaporation; eventually the drop vanishes at a finite-time singularity. Our theory, based on a first-principles hydrodynamic description, well reproduces the dynamics of $R$ and the value of $\theta$ during retraction.

\section{Key words:}

\section{Introduction}

The evaporation of liquid drops, in the form of dew, rain, or mist generated by breaking waves, must be accounted for accurately in the heat and mass balance of climate models. Evaporation is also important for industrial processes such as spray drying or ink jet printing. As a result, the evaporation of drop has attracted a great deal of attention over the past few years (for recent reviews see Cazabat \& Guena 2010; Erbil 2012; Larson 2014).

The two situations most studied are (i) the "coffee-stain" problem in which a drop is deposited on a rough substrate to which its contact line remains anchored during evaporation (Deegan et al. 1997, 2000) and (ii) drop of completely wetting liquids deposited on a perfectly smooth surface (Cachile et al. 2002a,b; Poulard et al. 2005; Shahidzadeh-Bonn et al. 2006). The latter problem, studied here, has attracted a great deal of attention since it is unclear why a completely wetting liquid exhibits a non-zero contact angle during evaporation (Elbaum et al. 1995; Bonn \& Meunier 1997). This problem is difficult because it involves diverging viscous stresses and evaporation rates, which need to be regularized to predict the motion (Bonn et al. 2009; Eggers \& Pismen 2010). In doing so, the shape of the drop is a priori unknown and has to be calculated; however this requires the prediction of the speed of the moving contact line, which is due to a complicated interplay between pinning, thermal activation and viscous dissipation (Snoeijer \& Andreotti 2013; Perrin et al. 2016). In addition, numerous secondary effects

$\dagger$ Email address for correspondence: E.A.M.Jambon-Puillet@uva.nl 


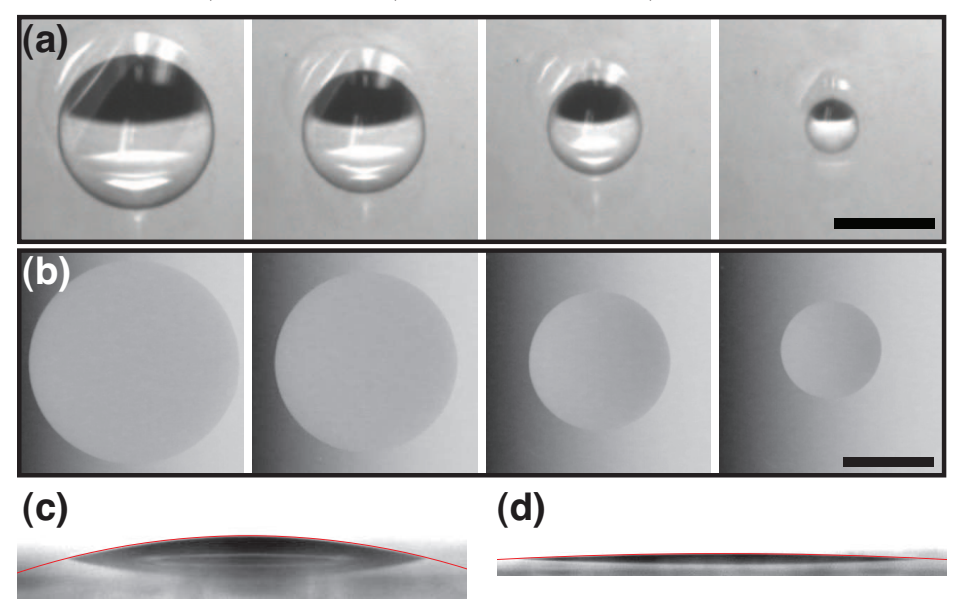

Figure 1. Top (a)-(b) and side (c)-(d) view of evaporating and spreading drops on clean glass surfaces. (a) Pentane drop, scale bar $5 \mathrm{~mm}, \mathrm{~d} t=6 \mathrm{~s}$. (b) Heptane drop, scale bar $2 \mathrm{~mm}$, $\mathrm{d} t=10 \mathrm{~s}$. (c) Peptane drop, $R=1.4 \mathrm{~mm}$. (d) Heptane drop, $R=2.6 \mathrm{~mm}$, the images include the drop's reflection on the solid surface, the red line is a spherical cap fit.

can arise from evaporation such as buoyant convection (Shahidzadeh-Bonn et al. 2006), Kelvin effects: curvature dependence of the equilibrium vapour pressure (Rednikov \& Colinet 2013; Janeček et al. 2013), or non-uniform temperatures. The latter may lead to Marangoni flows: surface flows driven by surface tension gradients (Hu \& Larson 2006).

Here we study the relative effect of evaporation and spreading systematically by placing completely wetting drops of alkanes (pentane $\left(\mathrm{C}_{5} \mathrm{H}_{12}\right)$ to nonane $\left(\mathrm{C}_{9} \mathrm{H}_{20}\right)$ ), whose volatility varies by two orders of magnitude, on a clean glass surface (see figure 1 (a)(b)). The perfectly circular drop shape indicates that contact line pinning is not important. Our drops are sufficiently small, so that convection in the gas phase is negligible, and the evaporation rate is limited by vapour diffusion into the surrounding gas phase. Moreover, our drops are very thin, which limits temperature gradients, especially for alkanes that do not evaporate too fast.

Previous studies have found that the contact angle of such a completely wetting but evaporating drop can be non-zero (Bourges-Monnier \& Shanahan 1995; Cachile et al. $2002 a, b$; Poulard et al. 2005; Shahidzadeh-Bonn et al. 2006; Lee et al. 2008). The interpretation of such a non-zero contact angle for a completely wetting liquid, which we denote by $\theta_{e v}$, is difficult, since it represents a fundamentally non-equilibrium situation. The presence of stress and evaporative singularities at the contact line, which need to be regularized on a microscopic scale, make the problem inherently multi-scale. A crude regularization as proposed by Poulard et al. (2005) allows to understand the formation of such an angle but its exact expression has remained a subject of debate (Eggers \& Pismen 2010; Morris 2014). The recent paper by Saxton et al. (2016) only considers partially wetting liquids (while ignoring pinning of the contact line). The time dependence of the drop radius is also intriguing. Since the fluid is wetting it starts to spread, but at some point, the evaporation starts to dominate, the drop retracts and $R$ eventually vanishes at a time $t_{0}$.

In this article, using the framework proposed by Eggers \& Pismen (2010), later developed by Morris (2014) we propose a simple parameter free model to describe the spreading dynamics and contact angle of evaporating drops of completely wetting liquids and make a direct comparison with experiments. 


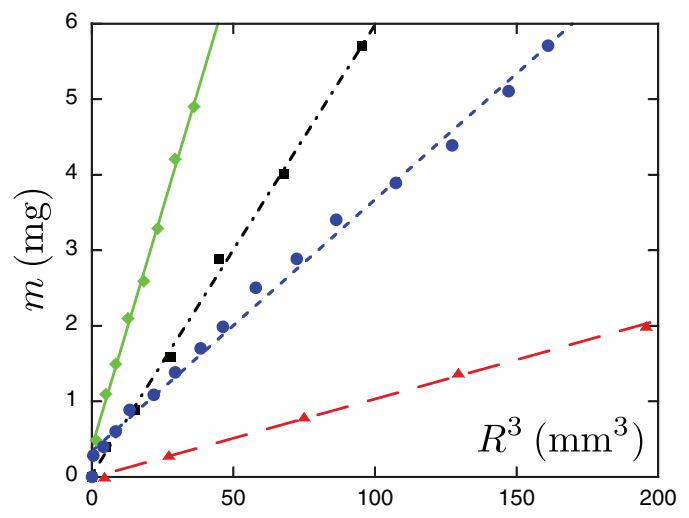

Figure 2. Mass of the drop vs. radius cubed; straight lines indicate a constant contact angle. Pentane (C5): green diamonds; hexane (C6): black squares; heptane (C7): blue circles; octane (C8): red triangles.

\section{Experimental set-up}

Experiments were performed at room temperature $T \approx 21^{\circ} \mathrm{C}$ by gently depositing a drop on a float glass surface using a microsyringe, and recording either its weight or shape using a precision balance and a drop shape analyser (Kruss Easydrop, see figure 1 (c)(d)). The alkanes used were ultra pure, from Sigma-Aldrich, the substrate were glass microscope slides (Menzel Gläser, $1 \mathrm{~mm}$ thick), cleaned with either sulfochromic acid or piranha solution. The equilibrium vapour pressure $P_{\text {sat }}$ of the different alkanes varies over two orders of magnitude, while keeping almost the same density $\rho$, surface tension $\gamma$ and viscosity $\eta$. The volume of the drops was about $1 \mu \mathrm{L}$. The largest Bond number was $B o=\rho g h R / \gamma \approx 0.2$ so gravity could be neglected ( $h$ is the drop height). The drop profile being well fitted by a spherical cap (figure 1 (c)(d)) the drop volume $V$, and apparent contact angle $\theta$ are calculated from the drop height $h$ and radius $R$ assuming a spherical cap profile.

\section{Result and discussion}

Figure 2 shows the mass $m$ of various alkane drops as a function of their radius cubed as they evaporate, giving a straight line. For a thin drop, $\theta=4 m /\left(\pi \rho R^{3}\right)$, so that the slope in figure 2 directly corresponds to the contact angle, which is seen to depend strongly on the chain length of the alkane, despite their similar interfacial properties. The macroscopic contact angle is thus controlled by evaporation rather than the wetting properties; due to their low surface tension in equilibrium the contact angle of all alkanes on the substrate is zero.

Turning to the drop dynamics, the simplest assumption is that to leading order the drop dynamics are unaffected by evaporation, which enters through the total mass balance only. Thus drop motion is described by Tanner's law (Bonn et al. 2009): $R \sim$ $V^{3 / 10}(\gamma t / \eta)^{1 / 10}$, but the total mass flux is proportional to the drop radius (Deegan et al. 2000):

$$
\dot{V}=-4 \beta R \text {. }
$$

Here $\beta$ is the evaporation parameter which can be approximated as $\beta=D\left(\rho_{\text {sat }}-\rho_{\infty}\right) / \rho$ for thin drops (Cazabat \& Guena 2010) with $\rho_{\text {sat }}$ the saturation vapour density, $\rho_{\infty}$ the vapour density far from the drop $\left(\rho_{\infty}=0\right.$ for alkanes) and $D$ the vapour diffusion coefficient. Solving the resulting differential equation for $V$, and substituting back into 


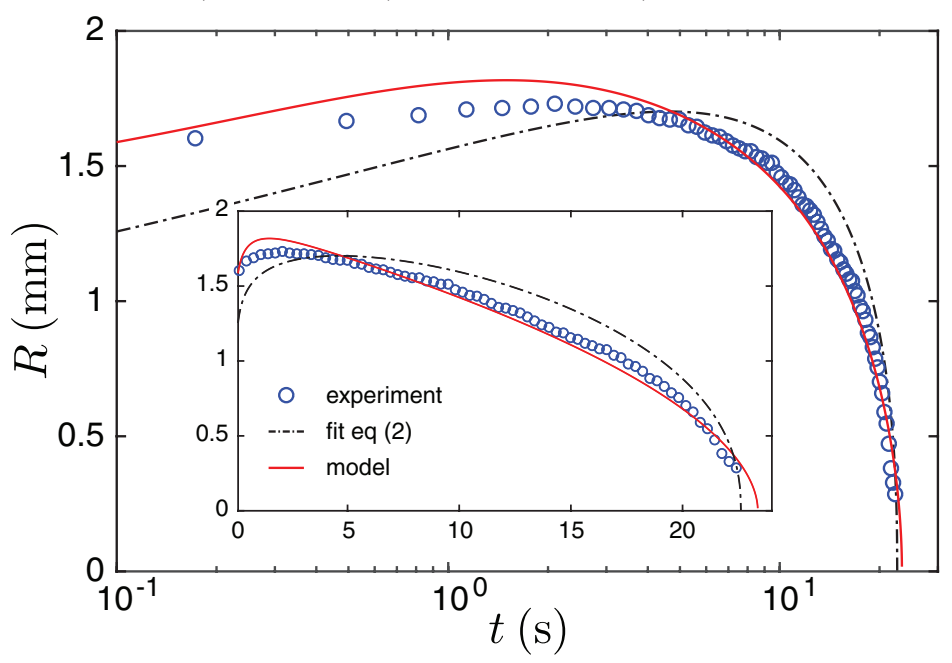

FiguRE 3. Spreading and evaporation of a $0.32 \mu \mathrm{L}$ heptane drop with a best fit of (3.2) as the dot-dashed line (prefactor $=0.36, t_{0}=22.6$ ), and our model as the red solid curve. (inset) Same data on a linear scale.

Tanner's law to find $R$, we find

$$
R \propto\left[t_{0}^{11 / 10}-t^{11 / 10}\right]^{3 / 7} t^{1 / 10}
$$

Figure 3 shows (3.2) as the dot-dashed line, with both a prefactor and $t_{0}$ as adjustable parameters. Clearly, this simple theory is unable to describe the drop dynamics satisfactorily, demonstrating that evaporation must be included into the description of the contact line dynamics itself, rather than including it phenomenologically.

To do better, one needs to solve the viscous flow problem in the drop coupled to the evaporation which is limited by the diffusion of vapour. For thin isothermal drops, the flow is simplified through the lubrication approximation and the flow profile is parabolic. The evolution of the drop shape is given by mass conservation (in axisymmetric coordinates),

$$
\frac{\partial h}{\partial t}+\frac{1}{r} \frac{\partial}{\partial r}\left(\frac{h^{3} r}{3 \eta} \frac{\partial p}{\partial r}\right)=-j_{e v}, \quad j_{e v}=-\frac{D}{\rho} \frac{\partial \rho_{v}}{\partial z},
$$

$p$ is the pressure driving the flow and $j_{e v}$ the local volume flux induced by the diffusion limited evaporation ( $\rho_{v}$ is the vapour density).

At the macroscopic scale, the pressure is simply the Laplace pressure and the vapour concentration is given by Laplace's equation $\nabla^{2} \rho_{v}=0$ with boundary condition $\rho_{v}=\rho_{\text {sat }}$ at the drop surface and $\rho_{v}=\rho_{\infty}$ far from the drop. Approximating the thin drop as a disc allows to compute the vapour field $\rho_{v}$ and the volume flux $j_{e v}=2 \beta /\left(\pi \sqrt{R^{2}-r^{2}}\right)$ (Jackson 1975). However, this macroscopic description suffers from the usual viscous stress divergence at the contact line, also present without evaporation (Bonn et al. 2009; Eggers \& Fontelos 2015). In addition, $j_{e v}$ is also singular at $r=R$ (the divergence persists for spherical caps with low contact angle (Deegan et al. 2000)). To deal with the problem one has to introduce microscopic effects to regularize the singularities.

A first attempt was made by Poulard et al. (2005) using scaling arguments. They introduce the distance from the contact line where van der Waals forces balance capillary forces and assume that the evaporation rate saturates below this scale. The resulting model being based on scaling arguments, Poulard et al. (2005) did not perform a direct 
comparison with experiments. Nonetheless, their model predicts power laws for $R(t)$ during the retraction stage that agree with the ones observed experimentally.

More recently, Eggers \& Pismen (2010) introduced van der Waals forces selfconsistently in the coupled problem through a disjoining pressure term $\Pi=A /\left(6 \pi h^{3}\right)=$ $\gamma a^{2} / h^{3}$ ( $A$ is the Hamaker constant, and $a$ is a microscopic length). A consequence is that far from the drop, (attractive) van der Waals interactions compete with evaporation to condensate a microscopic prewetting film whose thickness $h_{f}$ is given by a balance of evaporative to disjoining pressure $h_{f}^{3}=\gamma a^{2} /\left(\rho R_{s} T \ln \left(\rho_{\infty} / \rho_{\text {sat }}\right)\right)$ with $R_{s}$ the specific gas constant. In addition, Eggers \& Pismen (2010) included the Kelvin effect which takes the local curvature of the drop into account. They showed that taking these effects into account regularizes the evaporative singularity as it inhibits evaporation. Equation (3.3) then becomes:

$$
\begin{gathered}
\frac{\partial h}{\partial t}+\frac{\gamma}{\eta r} \frac{\partial}{\partial r}\left[\frac{h^{3} r}{3} \frac{\partial}{\partial r}\left(\frac{\partial^{2} h}{\partial r^{2}}+\frac{1}{r} \frac{\partial h}{\partial r}+\frac{a^{2}}{h^{3}}\right)\right]=-j_{e v} \\
j_{e v}=\frac{\beta}{r} \frac{\partial}{\partial r}\left[\int_{0}^{\infty} \mathcal{K}\left(r, r^{\prime}\right) \frac{\partial}{\partial r^{\prime}}\left(\frac{h_{f}}{h}\right)^{3} \mathrm{~d} r^{\prime}\right] .
\end{gathered}
$$

The kernel is given by

$$
\mathcal{K}\left(r, r^{\prime}\right)=\frac{2}{\pi} \begin{cases}r\left[\mathrm{~K}\left(r^{\prime} / r\right)-\mathrm{E}\left(r^{\prime} / r\right)\right], & r^{\prime}<r \\ r^{\prime}\left[\mathrm{K}\left(r / r^{\prime}\right)-\mathrm{E}\left(r / r^{\prime}\right)\right], & r^{\prime}>r\end{cases}
$$

where $\mathrm{K}$ and $\mathrm{E}$ are the complete elliptic integrals.

In the quasi-static limit, which means that when considering evaporation, the time derivative in (3.4) is neglected, Morris (2014) shows that the contact region can be described analytically in the case of vanishing $\mathscr{L}$. This allows for the exact computation of the cut-off length introduced by hand by Poulard et al. (2005) and the determination of the evaporative angle (Morris 2014, see appendix B for the derivation of the closed form solution presented below). The result is

$$
\theta_{e v}=k\left(\frac{\eta \beta}{\gamma a^{1 / 2} R^{1 / 2}}\right)^{1 / 3},
$$

where $k$ can be assumed constant and is given by

$$
\begin{gathered}
k=1.47758 \frac{2^{1 / 6}}{\pi^{1 / 3}} \mathrm{~W}\left(\frac{4}{\mathscr{L}^{3 / 2}}\right)^{1 / 6}, \\
\mathscr{L}=\frac{2^{2 / 3} \rho_{\text {sat }}^{4 / 3}(\eta \beta)^{16 / 9}}{\pi^{4 / 9} a^{26 / 9} R^{2 / 9} \gamma^{4 / 9}\left(R_{s} T \rho\left(\rho_{\text {sat }}-\rho_{\infty}\right)\right)^{4 / 3}},
\end{gathered}
$$

were $\mathrm{W}$ denotes the Lambert $\mathrm{W}$ function (see appendix $\mathrm{B}$ and table 2 for values of $\mathscr{L}$ and $k)$.

Motion is such that the apparent contact angle $\theta$ is driven toward $\theta_{e v}$ according to the Cox-Voinov law (Eggers \& Fontelos 2015):

$$
\dot{R}=\frac{\gamma}{9 B \eta}\left[\left(\frac{4 V}{\pi R^{3}}\right)^{3}-\theta_{e v}^{3}\right]
$$

where $B$ is the usual logarithmic molecular cut-off also present without evaporation (see appendix B).

The equations (3.1),(3.6),(3.8) derived here are the same as the scaling analysis 


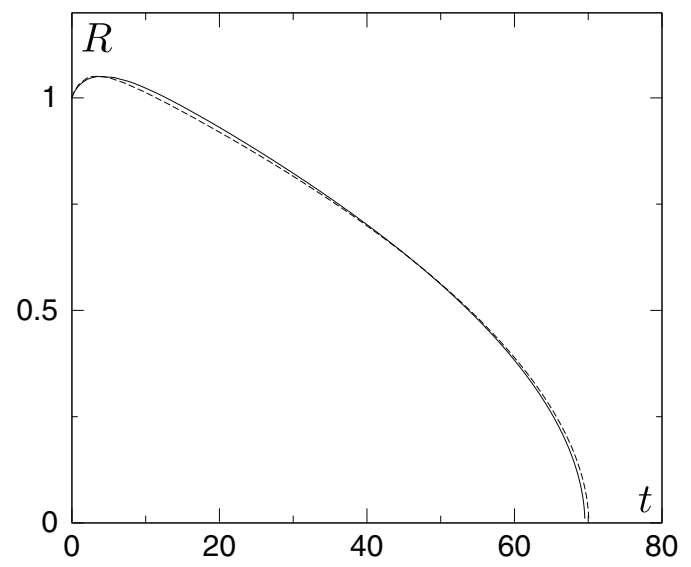

Figure 4. Dimensionless radius as a function of the dimensionless time for an evaporating drop, with $\beta=510^{-3}, a=10^{-3}$ and $h_{f}=10^{-4}$. The solid line is the simulation and the dashed line is theory $(B \approx 2.1$ with these parameters).

proposed in (Poulard et al. 2005); however because of the nature of their analysis, they were unable to calculate the prefactors and the discussion remained qualitative. Here we have done the full analysis; the evaporative cut-off is calculated by including the effect of disjoining pressure self consistently (Morris 2014).

We have simulated the complete equations of motion (3.4),(3.5) with parameters that lie in the quasi-static regime, and compared it to the model $(3.1),(3.6),(3.8)$ in figure 4 (lengths are rescaled with the initial drop radius $R_{0}$ and time with $\left.R_{0} \eta / \gamma\right)$. The model shows a very good agreement with the simulation with a fitted value $k=1.9$ close to the one predicted by equation (3.7): $k=1.69$.

Now, comparing the model to experimental data, we measure the evaporation parameter $\beta$ using (3.1) and estimate $a \approx 4 \AA$ using Lifshitz theory (Israelachvili 2011); $B$ in (3.8) is calculated from (B 7) and varies between 5.38 and 6.03 (table 2). The range being narrow we use the mean value 5.6 for all our experimental comparisons. Similarly we calculate the parameter $k$ in (3.6) from (3.7). It varies between 1.42 and 1.69 (table 2 ) and we use an intermediate value of 1.62 for all our experiments.

Figures 3 and 5 compare $R(t)$ from the model with the experimental data for various alkanes. We find excellent agreement for slowly evaporating alkanes: heptane to nonane, without any free parameters, cf. figure 5 (a). In addition, within the experimental accuracy, the macroscopic contact angle $\theta(t)$ is also well described by these equations, and approaches a constant steady state value at late times (inset figure 5 (a)). For pentane and hexane, for which evaporation is very rapid, the drop hardly spreads and the contact line recedes quickly (figure $5(\mathrm{~b})$ ). During the short spreading time, $\theta$ decreases significantly (inset of figure $5(\mathrm{~b})$ ); this means that $\partial h / \partial t$ is not small, and the quasistatic assumption used in the model is not valid. Moreover, the cooling due to evaporation increases with the evaporation rate $\beta$ and neglecting the temperature gradient and resulting Marangoni flows becomes incorrect (see appendix A for a critical discussion of the model's assumptions). As a result, the model is only able to reproduce these dynamics qualitatively, as it overestimates the spreading motion at short times.

As for the contact angle, the breakdown of the quasi-static and isothermal assumptions means that the measured angle does not necessarily converge to $\theta_{e v}$ given by (3.6). Nevertheless, the experimental contact angle $\theta$ reaches a steady-state value close to $\theta_{e v}$. We plot this value as a function of $\beta$ for the different alkanes in figure 6 . Within the 

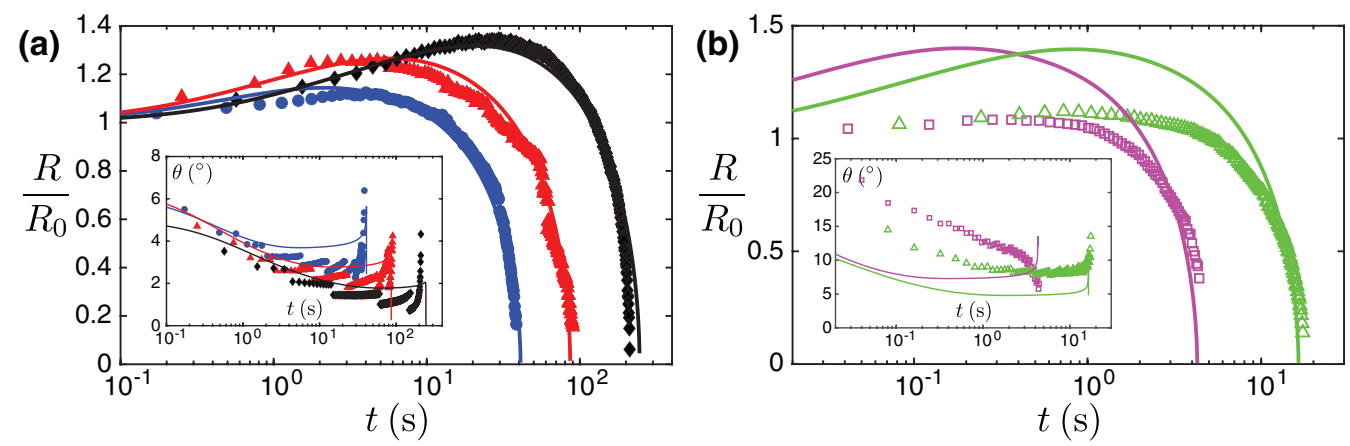

FiguRE 5. Dimensionless radius of spreading and evaporating alkane drops. (inset) Measured contact angle for the same data set, the uncertainties are not reported here for clarity (see figure 6). (a) Blue circles: heptane; red triangles: octane; black diamonds: nonane. (b) Pink open squares: pentane; green open triangles: hexane.

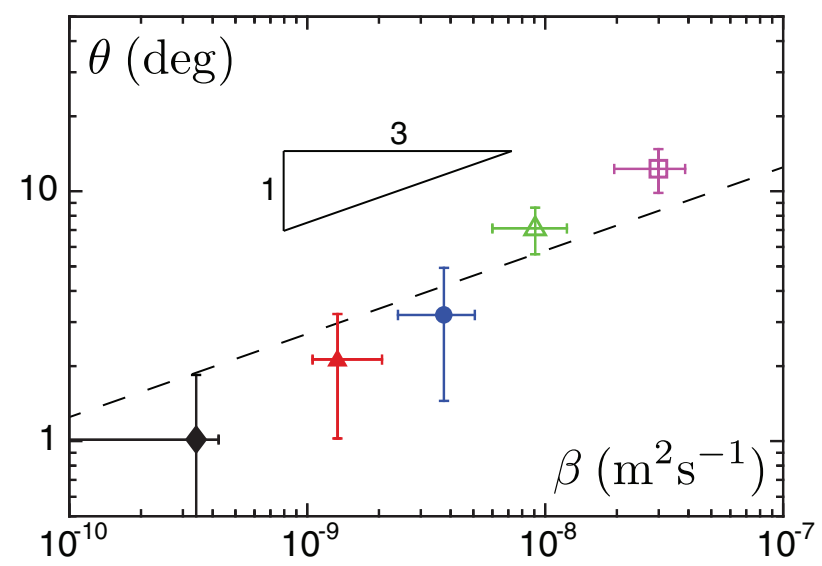

Figure 6 . Contact angle at late times $\theta$ as a function of the evaporation parameter $\beta$. The dashed line is equation (3.6), neglecting the small variations in the parameters for alkanes: $\theta_{e v}(\mathrm{rad})=46.88 \beta^{1 / 3}$.

experimental uncertainties (that become significant when the drop is very thin) one retrieves the $1 / 3 \mathrm{rd}$ power law predicted by (3.6) with the correct prefactor.

\section{Conclusion}

In summary, we studied the dynamics of perfectly wetting, volatile fluids on a solid substrate for a wide range of evaporation rates. Taking into account both spreading dynamics as well as using a consistent description of evaporation near the contact line, we were able to obtain a quantitative agreement between our parameter free model and experiments during both spreading and retraction phases for slowly evaporating alkanes. For very volatile liquids, the agreement is only qualitative as temperature gradients and dynamic effects, neglected in the model, become significant.

This work received a financial grant from the "French Agence Nationale de la Recherche"; project ANR-13-BS09-0026. 


\section{Appendix A}

In our model we assume the system to be isothermal (and thus without any Marangoni flows), the drop movement to be quasi-static and the transport of vapour to be purely diffusive (neglecting convection and kinetic effects). We will now discuss the validity of these assumptions using the dimensionless groups as proposed in Larson (2014).

Because the experiments are carried out in a box to limit air flows around the drop, the only source of convection in our experiment is the natural convection due to the buoyancy of the alkane vapour in the air. The Grashof number $G r$, which balances buoyancy with viscous forces, controls the strength of this natural convection. Assuming the gas to be ideal and the vapour concentration to be the saturation concentration, we have

$$
G r=\frac{\left(\rho_{\text {sat }}-\rho_{\infty}\right) g R^{3}}{\rho_{\text {air }} \nu_{\text {air }}^{2}},
$$

where $\rho_{\text {air }}$ is the air density and $\nu_{\text {air }}$ the air kinematic viscosity.

Kelly-Zion et al. (2011) studied the effect of natural convection in evaporating sessile drops with pinned contact line. They found the empirical relationship

$$
\dot{V}=-4 \beta R\left(1+0.310 G r^{0.216}\right),
$$

which differs significantly from the purely diffusive case if $0.310 G r^{0.216}>1$. We show in table 1 the Grashof number corresponding to our experiments (using $R_{0}$ for the drop radius). According to Kelly-Zion et al. (2011), the maximum convective contribution we can expect (as $R$ decreases after the spreading phase) ranges between 0.5 and 0.9 times the diffusive contribution, which is neither dominant nor negligible. Nonetheless our $\dot{V}=f(R)$ data are fairly linear, but with a coefficient $\beta$ somewhat higher than $D \rho_{\text {sat }} / \rho$, the value expected in the purely diffusive case (see table $1, \rho_{\infty}=0$ for alkanes). Although this is difficult to quantify given our experimental uncertainties, there might be a little bit of convection in some of our experiments. Though this is not the reason why the model fails to describe the dynamics for short alkanes. For hexane and pentane the discrepancies are small and replacing (3.1) by (A 1) does not improve the model significantly. Thus we keep the pure diffusion approximation in our model, but we use the measured $\beta$ directly instead of using the predicted value $D \rho_{\text {sat }} / \rho$.

If the diffusion of the vapour in the ambient gas is very fast, for instance under reduced pressure or if the ambient gas is pure vapour, then the evaporation can become affected by kinetic effects: the transfer of molecules from the liquid to the vapour at the interface (given by the Hertz-Knudsen relation). This effect reduces the concentration of vapour at the liquid-vapour interface and thus the overall evaporation rate. However, scaling arguments (Cazabat \& Guena 2010; Larson 2014) and numerical simulations (Semenov et al. 2012) have shown that this effect is negligible for common liquids in ambient air, except for microscopic droplets. Moreover, pure diffusion predicts our measured evaporation rate satisfactorily so we neglect interfacial kinetic effects.

The possible temperature gradients come from the heat loss due to latent heat of evaporation whose average rate is $\rho \dot{V} \Delta H_{v a p} / \pi R^{2}$, with $\Delta H_{v a p}$ the heat of vaporisation per unit mass. This flux must be balanced by steady-state heat conduction from the substrate of the order of $k_{l} \Delta T / h$ with $k_{l}$ the liquid thermal conductivity. Equating the two allows to evaluate the temperature gradient

$$
\Delta T \sim \frac{4 \rho \beta \Delta H_{v a p} h}{\pi k_{l} R} .
$$

For alkanes $\Delta H_{v a p} \approx 3.510^{5} \mathrm{~J} \mathrm{~kg}^{-1}$ and $k_{l} \approx 0.13 \mathrm{~W} \mathrm{~m}^{-1} \mathrm{~K}^{-1}$, the temperature gradients are thus directly proportional to $\beta$ times the drop aspect ratio $h / R$. As the drop aspect 


\begin{tabular}{ccccccccc} 
alkane & $\begin{array}{c}R_{0} \\
(\mathrm{~mm})\end{array}$ & $\begin{array}{c}h_{0} \\
(\mathrm{~mm})\end{array}$ & $\begin{array}{c}\beta \\
\left(10^{-9} \mathrm{~m}^{2} \mathrm{~s}^{-1}\right)\end{array}$ & $\begin{array}{c}D \frac{\rho_{\text {sat }}}{\rho} \\
\left(10^{-9} \mathrm{~m}^{2} \mathrm{~s}^{-1}\right)\end{array}$ & $\begin{array}{c}G r \\
(\mathrm{~K})\end{array}$ & $\begin{array}{c}\Delta T \\
\left.\frac{\partial h}{\partial t}\right|_{t=0} \\
\left(\mathrm{~mm} \mathrm{~s}^{-1}\right)\end{array}$ \\
\hline pentane & 1.27 & 0.238 & $29.9 \pm 9.6$ & 27.1 & 146 & 12.1 & 12000 & -0.68 \\
hexane & 1.68 & 0.211 & $9.07 \pm 3.2$ & 8.50 & 120 & 2.6 & 1800 & -0.34 \\
heptane & 2.37 & 0.128 & $3.75 \pm 1.3$ & 2.67 & 119 & 0.5 & 160 & -0.06 \\
octane & 2.12 & 0.120 & $1.34 \pm 0.5$ & 0.79 & 30 & 0.2 & 43 & -0.10 \\
nonane & 2.12 & 0.071 & $0.34 \pm 0.2$ & 0.17 & 8 & 0.02 & 3 & -0.01
\end{tabular}

TABle 1. Parameters used to asses the validity of our assumptions; the experimental values corresponds to the experiment presented in figure $5, D$ values at $T=22^{\circ}$ come from (Berezhnoi \& Semenov 1997; J. Beverley et al. 1999), Psat values from (Carruth \& Kobayashi 1973; J. Beverley et al. 1999).

ratio is larger for drops which evaporates faster, we immediately see they have the largest temperature gradient. These temperature gradients can induce Marangoni flows which appear for values of the Marangoni number

$$
M a=\frac{\mathrm{d} \gamma}{\mathrm{d} T} \frac{\Delta T R}{\alpha_{l} \eta}
$$

above a critical value $M a_{c} \sim 10^{2}$. Here $\alpha_{l}$ is the thermal diffusivity of the liquid and is of the order $10^{-7} \mathrm{~m}^{2} \mathrm{~s}^{-1}$ for alkanes while $\mathrm{d} \gamma / \mathrm{d} T \approx 10^{-4} \mathrm{~N} \mathrm{~m}^{-1}$. We evaluate both $\Delta T$ and $M a$ for our drops in table 1 using the initial values $h_{0}, R_{0}$ for the drop shape and our measured value of $\beta$. We see that the temperature gradient is very large for pentane drops and still significant for hexane drops. This produces Marangoni numbers well above the instability threshold for these drops. Thus our neglect of the Marangoni stress in the lubrication analysis partly explains the discrepancy between model and experiment for the spreading dynamics and evaporative angle. For heptane drops $M a \sim M a_{c}$, however, because the model is able to reproduce the experimental data and the evaluation of $\mathrm{Ma}$ is approximate, we believe these Marangoni effects are still negligible.

To evaluate the quasi-static assumption we calculate characteristic time scales of our drops and compare them to the evaporation duration $t_{0}$. The characteristic time for heat equilibration inside the drop is $t_{\text {heat }} \sim h_{0}^{2} / \alpha_{l}$. Because the initial drop height $h_{0}$ decreases with the chain length (see table 1 ), so does $t_{\text {heat }}$, which varies between $0.05 \lesssim t_{\text {heat }}(\mathrm{s}) \lesssim$ 0.5. The drying time $t_{0}$, however increases with the chain length $5 \lesssim t_{0}(\mathrm{~s}) \lesssim 200$. Since $t_{0}>>t_{\text {heat }}$, we can neglect the time dependence of the temperature in the drop. Similarly the characteristic time for the velocity inside the drop to reach a steady state is $t_{m o m} \sim h_{0}^{2} \rho / \eta$. It also decreases with the chain length of the alkane and varies between $510^{-3} \lesssim t_{\text {mom }}(\mathrm{s}) \lesssim 0.2$. Again, since the drying time $t_{0}$ is much larger, the quasistatic approach is in general valid. However, the most unfavourable cases are pentane and hexane. For these fast evaporating alkane the spreading motion is very fast, and the reversal of the contact line occurs at $t_{R_{\max }} \sim 0.5 \mathrm{~s}$. During the spreading motion the system has not yet had time to reach a steady state. At this early stage $\partial h / \partial t$ is significantly higher for pentane and hexane (see table 1) and cannot be neglected.

\section{Appendix B}

With a local analysis of the coupled equations (3.4) in the vicinity of the contact line and in the quasi-static limit, Morris (2014) predicted the macroscopic contact angle of 
the drop $\theta_{e v}$ in the asymptotic limit $\mathscr{L} \rightarrow 0$ (equation (6.3) of Morris (2014)) :

$$
\theta_{e v}=1.47758\left(\frac{2 \sqrt{2}}{\pi} \frac{\eta \beta}{\gamma a^{1 / 2} R^{1 / 2}}\right)^{1 / 3} \ell_{1}^{1 / 4} .
$$

Here $\mathscr{L}$ is the Laplace parameter, which is a dimensionless surface tension controlling the coupled problem:

$$
\mathscr{L}=\frac{2^{2 / 3} \rho_{\text {sat }}^{4 / 3}(\eta \beta)^{16 / 9}}{\pi^{4 / 9} a^{26 / 9} R^{2 / 9} \gamma^{4 / 9}\left(R_{s} T \rho\left(\rho_{\text {sat }}-\rho_{\infty}\right)\right)^{4 / 3}},
$$

and $\left\{\ell_{1}, h_{1}\right\}$ are the dimensionless location and height at which the capillary and disjoining pressures balance in the wetting film, respectively. These lengths are given by (see equation (5.10) of Morris (2014)):

$$
\mathscr{L} \ell_{1} h_{1}^{4}=1 \quad \text { and } \quad \ell_{1}=\left(\frac{3}{2} \ln h_{1}\right)^{2 / 3} .
$$

Eliminating $\ell_{1}$ from (B 3) we arrive at:

$$
\frac{4}{\mathscr{L}^{3 / 2}}=\frac{4}{\mathscr{L}^{3 / 2} h_{1}^{6}} \exp \left(\frac{4}{\mathscr{L}^{3 / 2} h_{1}^{6}}\right)
$$

which can be solved in terms of the Lambert W function, and we obtain:

$$
h_{1}=\left(\frac{4}{\mathscr{L}^{3 / 2} \mathrm{~W}\left(4 / \mathscr{L}^{3 / 2}\right)}\right)^{1 / 6}, \quad \ell_{1}=\left(\frac{\mathrm{W}\left(4 / \mathscr{L}^{3 / 2}\right)}{4}\right)^{2 / 3} .
$$

Replacing (B 5) in (B 1), we obtain the final closed form equation for the evaporative contact angle:

$$
\theta_{e v}=k\left(\frac{\eta \beta}{\gamma a^{1 / 2} R^{1 / 2}}\right)^{1 / 3} \quad \text { with } \quad k=1.47758 \frac{2^{1 / 6}}{\pi^{1 / 3}} \mathrm{~W}\left(\frac{4}{\mathscr{L}^{3 / 2}}\right)^{1 / 6} .
$$

We show in table 2 the values obtained for $\mathscr{L}$ and $k$ using (3.7) with the measured value of $\beta$ and $R_{0}$. Since $R$ varies during an experiment, $\mathscr{L}$ and thus $k$ are not strictly constant, yet their variations are small so we neglect them. For instance $1.910^{-3}<\mathscr{L}<3.010^{-3}$ and $1.60<k<1.62$ for the octane drop of figure 5 (the minimum recorded drop radius is $0.3 \mathrm{~mm})$. $\mathscr{L}$ ranges between $310^{-4}$ for nonane and $810^{-2}$ for pentane, which gives $k \approx 1.55$ (see table 2 ). To compute $k$ for the simulation we rescale length with $R_{0}$ and time with $R_{0} \eta / \gamma$ and rewrite (B 2) using dimensionless parameters :

$$
\mathscr{L}=\frac{2^{2 / 3} \beta^{16 / 9} h_{f}^{4}}{\pi^{4 / 9} a^{50 / 9} R^{2 / 9}} .
$$

In doing so we have used the linearised definition of $h_{f}$ as in Eggers \& Pismen (2010) which strictly speaking is not correct when $\rho_{\infty}=0$. The resulting prefactor $k=1.69$ is very close to experimental ones despite the very different values of the parameters.

Equation (B 6) predicts the experimental steady state contact angle quantitatively, except for pentane and hexane (see figure 5 (b)), for which a discrepancy larger than the uncertainties starts to appear. As discussed above some of the model's assumption break down for these drops, the isothermal assumption is incorrect and the quasi-static assumption becomes doubtful. We can also notice that $\mathscr{L}$ is much larger such that the limit $\mathscr{L} \rightarrow 0$ might not be reached. 
pentane hexane heptane octane nonane

\begin{tabular}{cccccc}
\hline $\mathrm{a}(\AA)$ & 4.29 & 3.96 & 3.95 & 3.85 & 3.78 \\
$\mathscr{L}$ & $8.010^{-2}$ & $1.810^{-2}$ & $6.510^{-3}$ & $2.010^{-3}$ & $3.010^{-4}$ \\
$k$ & 1.42 & 1.51 & 1.57 & 1.62 & 1.69 \\
$B$ & 5.38 & 5.75 & 5.58 & 6.03 & 5.52
\end{tabular}

TABle 2. Parameters deduced from the model for figure 5.

As in all moving contact line problems, the viscous stress in the vicinity of the contact line must be regularized in order to predict the drop radius as a function of time $R(t)$. This is usually done by introducing a cut-off length, which results in a logarithmic prefactor $B$ in the equation of motion in the contact line (de Gennes 1985; Bonn et al. 2009). In the very beginning of our experiment, spreading is dominant and the drop moves over a prewetting film. We thus use the cut-off length derived for spreading drops without evaporation (Bonn et al. 2009; Eggers \& Fontelos 2015):

$$
B=\ln \left(\frac{R}{1.38 e^{2} a}\left(\frac{\eta \dot{R}}{\gamma}\right)^{2 / 3}\right)
$$

and consider it constant throughout the experiment for simplicity (although it was not derived for an evaporating receding contact line). Table 2 shows the values of $B$ we obtain using the initial values of the experiments.

\section{REFERENCES}

Berezhnoi, A. N. \& Semenov, A. V. 1997 Binary diffusion coefficients of liquid vapors in gases. Begell House New York.

Bonn, D., Eggers, J., Indekeu, J., Meunier, J. \& Rolley, E. 2009 Wetting and spreading. Rev. Mod. Phys. 81, 739-805.

Bonn, D. \& Meunier, J. 1997 Comment on "evaporation preempts complete wetting". EPL (Europhysics Letters) 39 (3), 341.

Bourges-Monnier, C. \& Shanahan, M. E. R. 1995 Influence of evaporation on contact angle. Langmuir 11 (7), 2820-2829.

Cachile, M., BÉnichou, O. \& Cazabat, A. M. $2002 a$ Evaporating droplets of completely wetting liquids. Langmuir 18 (21), 7985-7990.

Cachile, M, Bénichou, O., Poulard, C. \& Cazabat, A. M. $2002 b$ Evaporating droplets. Langmuir 18 (21), 8070-8078.

Carruth, G. F. \& Kobayashi, R. 1973 Vapor pressure of normal paraffins ethane through n-decane from their triple points to about $10 \mathrm{~mm}$ mercury. J. Chem. Eng. Data 18 (2), $115-126$.

Cazabat, A. M. \& Guena, G. 2010 Evaporation of macroscopic sessile droplets. Soft Matter 6, 2591-2612.

Deegan, R. D., Bakajin, O., Dupont, T. F., Huber, G., Nagel, S. R. \& Witten, T. A. 1997 Capillary flow as the cause of ring stains from dried liquid drops. Nature $\mathbf{3 8 9}$ (6653), 827-829.

Deegan, R. D., Bakajin, O., Dupont, T. F., Huber, G., Nagel, S. R. \& Witten, T. A. 2000 Contact line deposits in an evaporating drop. Phys. Rev. E 62, 756-765.

Eggers, J. \& Fontelos, M. A. 2015 Singularities: formation, structure, and propagation. Cambridge University Press.

Eggers, J. \& Pismen, L. M. 2010 Nonlocal description of evaporating drops. Phys. Fluids 22 (11), 112101. 
Elbaum, M., Lipson, S. G. \& Wettlaufer, J. S. 1995 Evaporation preempts complete wetting. EPL (Europhysics Letters) 29 (6), 457.

ErBiL, H. Y. 2012 Evaporation of pure liquid sessile and spherical suspended drops: A review. Adv. Colloid Interface Sci. 170 (12), $67-86$.

De Gennes, P. G. 1985 Wetting: statics and dynamics. Rev. Mod. Phys. 57, 827-863.

Hu, H. \& Larson, R. G. 2006 Marangoni effect reverses coffee-ring depositions. J. Phys. Chem. B 110 (14), 7090-7094.

IsRaElachVili, J. N. 2011 Intermolecular and surface forces. Academic press.

J. Beverley, K, H. Clint, J \& D. I. Fletcher, P 1999 Evaporation rates of pure liquids measured using a gravimetric technique. Phys. Chem. Chem. Phys. 1, 149-153.

JaCkson, J. D. 1975 Classical Electrodynamics. Wiley, New York.

Kelly-Zion, P. L., Pursell, C. J., Vaidya, S. \& Batra, J. 2011 Evaporation of sessile drops under combined diffusion and natural convection. Colloids Surf., A 381 (13), 31 - 36.

Larson, R. G. 2014 Transport and deposition patterns in drying sessile droplets. AIChE Journal 60 (5), 1538-1571.

Lee, K. S., Cheah, C. Y., Copleston, R. J., Starov, V. M. \& Sefiane, K. 2008 Spreading and evaporation of sessile droplets: Universal behaviour in the case of complete wetting. Colloids Surf., A 323 (13), 63 - 72.

MorRIs, S. J. S. 2014 On the contact region of a diffusion-limited evaporating drop: a local analysis. J. Fluid Mech. 739, 308-337.

Perrin, H., Lhermerout, R., Davitt, K., Rolley, E. \& Andreotti, B. 2016 Defects at the nanoscale impact contact line motion at all scales. Phys. Rev. Lett. 116, 184502.

Poulard, C., Guéna, G., Cazabat, A. M., Boudaoud, A. \& Ben Amar, M. 2005 Rescaling the dynamics of evaporating drops. Langmuir 21 (18), 8226-8233.

Rednikov, A. \& Colinet, P. 2013 Singularity-free description of moving contact lines for volatile liquids. Phys. Rev. E 87, 010401.

Saxton, M. A., Whiteley, J. P., Vella, D. \& Oliver, J. M. 2016 On thin evaporating drops: When is the $d^{2}$-law valid? Journal of Fluid Mechanics 792, 134167.

Semenov, S., Starov, V. M., Rubio, R. G. \& Velarde, M. G. 2012 Computer simulations of evaporation of pinned sessile droplets: Influence of kinetic effects. Langmuir 28 (43), $15203-15211$.

Shahidzadeh-Bonn, N., Rafai, S., Azouni, A. \& Bonn, D. 2006 Evaporating droplets. J. Fluid Mech. 549, 307-313.

Snoeiser, J. H. \& Andreotti, B. 2013 Moving contact lines: scales, regimes, and dynamical transitions. Annu. Rev. Fluid Mech 45, 269.

Janeček, V., Andreotti, B., PražÁk, D., Bárta, T. \& Nikolayev, V. S. 2013 Moving contact line of a volatile fluid. Phys. Rev. E 88, 060404. 